\title{
Diabetic ketoacidosis: Should current management include subcutaneous insulin injections?
}

\author{
Rocio Gavidia Quezada MD, Hawa Edriss MD
}

\begin{abstract}
Diabetic ketoacidosis is a well-known acute complication in patients with both type 1 and type 2 diabetes mellitus. Although mortality has decreased considerably, it remains an important cause for admission to intensive care units. Medical management includes intravenous fluid therapy, insulin, correction of electrolyte abnormalities, and addressing the precipitating factor which in most cases is infection or non-compliance with insulin therapy. Usually patients with diabetic ketoacidosis are admitted to the intensive care unit for continuous infusion of insulin; however, the development of rapid acting insulin analogues has made it possible to treat mild to moderate diabetic ketoacidosis with subcutaneous insulin. Although studies using subcutaneous insulin include only a small number of patients, this approach seems as effective as intravenous insulin infusions in patients with mild to moderate diabetic ketoacidosis. Diabetic education and close follow-up for patients admitted for diabetic ketoacidosis remain essential to avoid recurrence and readmissions.
\end{abstract}

Keywords: Diabetic ketoacidosis, acute complication in diabetes, rapid acting insulin analogues, subcutaneous insulin in diabetic ketoacidosis

\section{INTRODUCTION}

Diabetic ketoacidosis (DKA) is a well-known acute complication in patients with both type 1 and type 2 diabetes. This condition results from a relative or absolute insulin deficiency combined with counter-regulatory hormone excess: glucagon, catecholamines, cortisol, and growth hormone. ${ }^{1}$ Diabetic ketoacidosis can be life threatening, but mortality rates have fallen since 1980, according to the National Diabetes Surveillance Program of the CDC. ${ }^{2}$ Mortality is usually related to associated comorbidities rather than hyperglycemia and ketoacidosis. ${ }^{3}$

Corresponding author: Rocio Gavidia

Contact Information: Rocio.gavidia@ttuhsc.edu

DOI: $10.12746 /$ swrccc.v5i19.389
Diabetic ketoacidosis is defined by the presence of acidosis (serum bicarbonate $<15$ and/or $\mathrm{pH}<7.3$ ), ketosis (ketonemia $>3.0 \mathrm{mmol} / \mathrm{L}$ or ketonuria), and hyperglycemia with blood glucose $>250 \mathrm{mg} / \mathrm{dl}$ or known diabetes mellitus. ${ }^{4}$ Although hyperglycemia is usually present in DKA, euglycemic DKA has been reported in patients with type 1 diabetes who were vomiting, were fasting, were pregnant, or had been treated with insulin prior to presentation. ${ }^{5,6}$ Euglycemic diabetic ketoacidosis has also been recently reported in patients using sodium-glucose cotransporter 2 inhibitors ${ }^{7}$, particularly in patients with type $1 \mathrm{DM}^{8,9}$

DKA can be classified depending on severity of presentation into mild, moderate, or severe categories.

\section{Pathophysiology}

Diabetic ketoacidosis is precipitated by inadequate levels of plasma insulin due to insulin deficiency 
Table DKA classification

\begin{tabular}{|l|c|c|c|}
\hline & Mild & Moderate & Severe \\
\hline Plasma glucose $(\mathrm{mg} / \mathrm{dL})$ & $>250$ & $>250$ & $>250$ \\
\hline Arterial $\mathrm{pH}$ & $7.25-7.30$ & $7.00-7.24$ & $<7.00$ \\
\hline Serum bicarbonate meq/L & $15-18$ & $10-<15$ & $<10.0$ \\
\hline Urine ketones & + & + & + \\
\hline Serum ketones & + & + & + \\
\hline Effective serum osmolarity & Variable & Variable & $>12$ \\
\hline Anion gap & $>10$ & $>12$ & Stupor/coma \\
\hline Mental status & Alert & Alert/drowsy & \\
\hline
\end{tabular}

Adapted from ADA guidelines for DKA management. ${ }^{10}$

or increased insulin requirements, along with excess of counter regulatory hormones. Both insulin deficiency and glucagon excess are necessary for DKA to develop. The decreased ratio of insulin to glucagon promotes gluconeogenesis, glycogenolysis, and ketone body formation in the liver and increases free fatty acid and amino acids delivery from fat and muscle to the liver. Insulin deficiency also reduces levels of the glucose transporter GLUT4, which impairs glucose uptake into skeletal muscle and fat and reduces intracellular glucose metabolism. Ketosis results from an increase in free fatty acid release from adipocytes and a shift toward ketone body synthesis in the liver. At physiologic $\mathrm{pH}$, ketone bodies exist as ketoacids, which are neutralized by bicarbonate. As bicarbonate stores are depleted, a metabolic acidosis develops. ${ }^{1}$

\section{Medical management}

The principles in the management of DKA are:

- IV fluids and correction of electrolyte abnormalities,

- Insulin therapy,

- Correction of the underlying precipitating factor

IV FLUIDS

Initial fluid therapy should expand intravascular and extravascular volume and restore renal perfusion. In the absence of heart failure, isotonic saline $(0.9 \%$
$\mathrm{NaCl}$ ) should be infused prior to insulin administration at a rate of $15-20 \mathrm{ml} / \mathrm{kg} / \mathrm{hr}$ or greater during the $1 \mathrm{st}$ hour $\left(-1-1.5 \mathrm{~L}\right.$ in the average adult).$^{10}$ Fluid administration in the first hour of therapy before insulin administration has the following advantages: 1) it allows time to obtain a serum potassium level on presentation, 2) it corrects hypotension, which may increase if insulin is used without hydration, 3) it improves insulin action and may reduce the concentration of counter regulatory hormones and hyperglycemia. ${ }^{11,12}$

ADA guidelines recommend that the subsequent choice for fluid replacement depends on the state of hydration, serum electrolyte levels, and urinary output. In general, $0.45 \% \mathrm{NaCl}$ infused at $4-14 \mathrm{ml} / \mathrm{kg} / \mathrm{hr}$ is appropriate if the corrected serum sodium is normal or elevated; $0.9 \% \mathrm{NaCl}$ at a similar rate is appropriate if corrected serum sodium is low. Martin et al prospectively studied the effects of hypotonic, isotonic, and hypertonic fluids in patients with severe DKA. This study reported no significant difference in the volume of fluid retained with the different solutions; however, hypertonic fluids increased the frequency of hypertonicity, hypernatremia, and hyperchloremia. ${ }^{13}$ In addition, some patients treated with hypotonic fluids developed diuresis; hence, rapid repletion of the plasma and extracellular volume with isotonic fluids is indicated in patients with DKA. ${ }^{3}$ In patients with renal or cardiac dysfunction serum osmolality, mental status, and cardiac and renal status should be monitored frequently during IV fluid resuscitation to avoid iatrogenic fluid overload. After the blood glucose level 
reaches $<250 \mathrm{mg} / \mathrm{dL}$, IV fluids should be switched to $5 \%$ dextrose with $0.45-0.75 \% \mathrm{NaCl}^{10}$

\section{INSULIN THERAPY}

ADA guidelines recommend starting regular insulin with an initial IV bolus at $0.15 \mathrm{U} / \mathrm{kg}$ followed by a continuous infusion at a dose of $0.1 \mathrm{U} / \mathrm{kg} / \mathrm{hr}$. Glucose should fall by $50 \mathrm{mg} / \mathrm{dl} / \mathrm{hr}$. If the blood glucose does not decrease at this rate, hydration status should be rechecked and insulin infusion can be increased to reach a steady decline in blood glucose of $50-75 \mathrm{mg} / \mathrm{h}$. When the plasma glucose falls to $250 \mathrm{mg} / \mathrm{dl}$, the insulin infusion rate can be decreased to $0.05-0.1 \mathrm{U} / \mathrm{kg} / \mathrm{hr}$. The rate of insulin administration may need to be adjusted to maintain the above glucose values until acidosis resolves.

A new approach of insulin therapy has been recently studied. The fact that patients in diabetic ketoacidosis are admitted to ICUs for continuous insulin infusions and monitoring has led investigators to look for alternative strategies for treatment. A prospective randomized controlled trial by Doshi et al assigned 20 patients to insulin infusion at $0.1 \mathrm{U} / \mathrm{kg} / \mathrm{hr}$ at a set rate and 20 patients to IV insulin $0.1 / \mathrm{kg} / \mathrm{hr}$ plus insulin Glargine at $0.3 \mathrm{U} / \mathrm{kg}$ at the time of diagnosis. ${ }^{14}$ The primary end point was time to closure of the anion gap (TCAG). Upon closure of the anion gap, the control group received subcutaneous long acting insulin and IV insulin was continued for 2 more hours. In the experimental group, the insulin infusion was discontinued and the patients received long acting insulin $24 \mathrm{hrs}$ after the initial dose. The estimated mean TCAG was 10.2 hrs in the experimental group and $11.6 \mathrm{hrs}$ in the control group $(p=0.63)$. Hospital length of stay was also slightly shorter in the experimental group (3.9 days) vs. the control group (4.6 days) $(p=0.66)$. There were no significant differences in outcomes between these two study arms.

Researchers have also started to study rapid acting insulin analogues as an alternative to the standard IV regular insulin continuous infusions in patients with diabetic ketoacidosis. Umpierrez et al studied 45 patients with DKA and randomly assigned them to 3 groups: 1) initial subcutaneous injection of $0.3 \mathrm{U} /$ $\mathrm{kg}$ of insulin aspart, followed by $0.1 \mathrm{U} / \mathrm{kg}$ every hour until resolution of DKA, 2) initial SC injection of 0.3 $\mathrm{U} / \mathrm{kg}$ of aspart followed by a dose of $0.2 \mathrm{U} / \mathrm{kg} 1$ hour later and every 2 hours thereafter until blood glucose was $250 \mathrm{mg} / \mathrm{dl}$. The dose was then reduced to $0.1 \mathrm{U} / \mathrm{kg}$ every 2 hours until resolution of DKA, 3) initial bolus of $0.1 \mathrm{U} / \mathrm{kg}$ of regular insulin followed by a continuous infusion at $0.1 \mathrm{U} / \mathrm{kg} / \mathrm{hr}$ until blood glucose was $250 \mathrm{mg} / \mathrm{dL}$. The infusion rate was then decreased to $0.05 \mathrm{U} / \mathrm{kg} / \mathrm{hr}$ until resolution of DKA. After comparing the three groups, the study found that the duration of treatment until resolution of hyperglycemia and DKA were similar among the three groups. The first group took on average $6.9 \mathrm{hrs}$ of therapy until blood glucose was below 250 compared to $6.1 \mathrm{hrs}$ in the second group and $7.1 \mathrm{hrs}$ in the IV continuous insulin infusion (third group). Duration of therapy until resolution of DKA was 10 hours in the first group, 10.7 hours in the second group, and 11 hours in the third group. ${ }^{15}$

Erso'z et al compared subcutaneous Lispro to regular insulin in 20 patients with mild to moderate DKA. Patients were randomized into two groups: Both groups received an initial bolus of $0.15 \mathrm{U} / \mathrm{kg}$ of IV regular insulin. Group L was then given $0.075 \mathrm{U} / \mathrm{kg}$ of subcutaneous Lispro every hour; Group R was treated with a continuous infusion of IV regular insulin. Time to achieve a glucose level of less than $200 \mathrm{mg} / \mathrm{dL}$ was 9.4 hours in the $L$ group and 12.7 hours in the $R$ group. B-hydroxybutyrate was less than $0.6 \mathrm{mEq}$ in $11.2 \mathrm{hrs}$ in the $L$ group vs. 15.3 hrs in the $R$ group. ${ }^{16}$

These studies suggest the following conclusions:

1. Duration of therapy until resolution of DKA in hours is 11-12 hrs $\pm 2 \mathrm{hrs}$ in the IV insulin infusion groups and 10-12 hrs $\pm 2 \mathrm{hrs}$ in the groups treated with SC insulin analogues.

2. Although the reported studies involved a small number of patients, treatment with subcutaneous insulin analogues appears to be effective in treating mild to moderate DKA patients.

It is important to understand that this type of treatment for DKA patients requires trained personnel in a step-down or regular floor units and very frequent subcutaneous injections which may not be practical on busy in-patient services. Although the studies 
discussed above had a relatively small number of patients, the recommendation for using subcutaneous insulin for carefully selected patients is already included in the most recent publication of the ADA for Diabetes Care in the Hospital. ${ }^{19}$ This approach has not been studied in patients with severe DKA and, therefore, is discouraged.

\section{ELECTROLYTE ABNORMALITIES}

\section{Potassium}

Serum potassium is frequently elevated in patients with diabetic ketoacidosis. This is caused by the extracellular shift of potassium in exchange for the hydrogen ions accumulated in acidosis and by the release of potassium from cells caused by glycolysis, insulin deficiency, and hyperosmolality. ${ }^{4}$ Treatment with insulin will shift potassium into the cell, causing a rapid decrease in potassium levels. ADA guidelines recommend that an insulin infusion should not be started if potassium levels are $<3.3 \mathrm{mEq} / \mathrm{l}$. If this is the case, potassium should be replaced before starting insulin treatment. When serum potassium levels are below $5.5 \mathrm{mEq} / \mathrm{l}$, maintenance potassium of $20 \mathrm{mEq}$ can be added to each liter of IV fluids to keep concentrations within the normal range and avoid hypokalemia.

\section{Phosphate}

Serum phosphate is lost by diuresis in diabetic ketoacidosis. Levels may be either normal or high upon presentation due to insulin deficiency. The level will start to decrease as soon as insulin treatment is established. ${ }^{4}$ Studies have failed to show any benefit with phosphate replacement in clinical outcomes in DKA. Overcorrection of phosphate can lead to severe hypercalcemia. Careful replacement may be indicated in patients with cardiac dysfunction, anemia, and respiratory depression and in patients with serum concentrations less than $0.32 \mathrm{mmol} / \mathrm{L}^{17}$

\section{BicARBonATE}

The use of bicarbonate therapy in patients with DKA is controversial. Some experts recommend the administration of $100 \mathrm{mmol}$ of sodium bicarbonate to patients with a $\mathrm{pH}<6.9 .{ }^{10}$ However, several studies have shown no benefit with the administration of bicarbonate. In a recent retrospective study, Duhon et al found no benefit in administering bicarbonate in patients with severe DKA with $\mathrm{pH}<6.9$. This study included 86 patients; 44 received IV bicarbonate therapy and 42 did not. The study concluded that the times to resolution of acidosis were similar in the two groups ( $8 \mathrm{hrs}$ vs. $8 \mathrm{hrs}, \mathrm{p}=0.72$ ). Additionally, patients who received IV bicarbonate also received slightly more insulin in the first 12 hrs of hospital stay (48 U vs. $44 U, p=0.05$ ) and first 24 hrs (100 U vs. $86 \cup \mathrm{u}=0.04)^{18 .}$ A prospective randomized study of 21 patients failed to show beneficial or adverse effects on morbidity and mortality with bicarbonate therapy in patients with DKA whose admission arterial $\mathrm{pH}$ was between 6.9-7.1. ${ }^{20}$ Patients with very low $\mathrm{pH}$ values may benefit from partial correction of their $\mathrm{pH}$. However, bicarbonate can cause hypokalemia, cerebral edema, and paradoxical central nervous system acidosis.

\section{CoRRECTION OF THE UNDERLYING ETIOLOGY}

The most common precipitating factors are infection and inadequate insulin treatment as a result of non-compliance, especially in young patients. Other factors include alcohol abuse, pancreatitis, myocardial infarction, trauma, drugs, and new onset diabetes, especially in patients with type 1 diabetes. Diabetic ketoacidosis has also been reported with mismanagement of insulin pumps or leakage of the infusion system. ${ }^{4}$

\section{RESOLUTION}

The resolution of DKA is determined by:

1. Blood glucose $<200-250 \mathrm{mg} / \mathrm{dL}$

2. Serum bicarbonate $>18 \mathrm{mEq} / \mathrm{L}$

3. Venous $\mathrm{pH}>7.3$

Blood glucose levels alone should not be used as an indicator for the resolution of DKA since ketonemia takes longer to resolve than hyperglycemia. After 
diabetic ketoacidosis resolves, IV insulin and fluid replacement should be continued if the patient is NPO. When the patient is able to eat, a combination of short or rapid acting insulin with intermediate or long acting insulin can be started. Intravenous insulin infusion should be continued 1-2 hrs after starting subcutaneous insulin to avoid rebound ketoacidosis. ${ }^{10}$ To transition to subcutaneous insulin it is recommended that $60-80 \%$ of the daily infusion should be administered as basal insulin. ${ }^{19}$ These patients need close follow-up in outpatient clinics and diabetic education.

Article citation: Gavidia Quezada R, Edriss H. Diabetic ketoacidosis: should current management include subcutaneous insulin injection? The Southwest Respiratory and Critical Care Chronicles 2017; 5(19): 6-10

From: Department of Internal medicine at Texas Tech University Health Sciences Center in Lubbock, TX

Submitted: $11 / 15 / 2016$

Accepted: 4/9/2017

Reviewer: Jose Beceiro MD

Conflicts of interest: none

\section{REFERENCES}

1. Powers AC. Harrison's Principles of Internal Medicine 17th edition, Chapter 338, Diabetes Mellitus, Diabetes Ketoacidosis Pathophysiology, p 2283.

2. Wang J, Williams DE, Narayan KM, Geiss LS. Declining death rates from hyperglycemic crisis among adults with diabetes, US 1985-2002. Diabetes Care 2006 Sep; 29(9):2018-22.

3. Nyenwe EA, Kitabchi AE. The Evolution of diabetic ketoacidosis: An update of its etiology, pathogenesis and management. Division of Endocrinology, Diabetes and Metabolism, University of Tennessee Health Science Center. https://doi. org/10.1016/j.metabol.2015.12.007. Published: 2016-04.

4. Dhatariya K. Diabetic emergencies. Diabetic Ketoacidosis and Hyperosmolar Crisis in Adults. Medicine; 42 (12): p723-726. [published online: October 20, 2014].

5. Munro JF, Cambell IW, McCuish AC et al. Euglycemic diabetic ketoacidosis. Br. Med J 1973; 2(5866):578-80.

6. Burge MR, Hardy KJ, Schade DS. Short-term fasting is a mechanism for the development of euglycemic ketoacidosis during periods of insulin deficiency. J Clin Endocrinol Metab 1993; 76(5):1192-8.
7. Juarez Ramirez Tello E, Orellana-Barrios M, Nugent K. Sodium-glucose co-transporter 2 inhibitor induced diabetic ketoacidosis in a type 2 diabetic patient. Amer J Med Sci; 351(6): 634-635.

8. Peters AL, Buschur EO, Buse JB, et al. Euglycemic diabetic ketoacidosis: A potential complication of treatment with sodium-glucose cotransporter 2 inhibition. Diabetes Care 2015 Sep; 38(9): 1687-1693.

9. Taylor SI, Blau JE, Rother KI. Perspective: SGLT2 inhibitors may predispose to ketoacidosis. J Clin Endocrinol Metab 2015 Aug; 100 (8):2849-52.

10. Hyperglycemic Crises in Diabetes. American Diabetes Association. Diabetes Care 2004. Jan; 27(Supplement 1): s94-s102.

11. Bratusch-Marrain PR, Komajati M, Waldhausal W. The effect of hyperosmolarity on glucose metabolism. Pract Cardiol 1985; 11:153-63.

12. Waldhausl W, Kleinberger G, Korn A, et al. Severe hyperglycemia: effects of rehydration on endocrine derangements and blood glucose concentration. Diabetes 1979; 28:577-84.

13. Martin HE, Smith K, Wilson ML. The fluid and electrolyte therapy of severe diabetic acidosis and ketosis; a study of twenty-nine episodes (twenty-six patients). Am J Med 1958; 24:376-89.

14. Doshi P, Potter A, De Los Santos D, Banuelos R, Darger $\mathrm{B}$, Chathampally Y. Prospective randomized trial of insulin glargine in acute management of diabetic ketoacidosis in the emergency department: a pilot study. Academic Emergency Medicine 2015; 22:658-662.

15. Umpierrez G, Cuervo R, Karabell A, Latif K, Freire A, Kitabchi A. Treatment of diabetic ketoacidosis with subcutaneous insulin aspart. Diabetes Care 2004 Aug; 27(8): 18731878. http://dx.doi.org/10.2337/diacare.27.8.1873

16. Ersöz HO, Ukinc K, Köse M, et al. Subcutaneous Lispro and intravenous regular insulin treatments are equally effective and safe for the treatment of mild and moderate diabetic ketoacidosis in adult patients. Int J Clin Pract 2006; 60:429-33.

17. Miller DW, Slovis CM. Hypophosphatemia in the emergency department therapeutics. Am J Emerg Med 2000; 18:457-61.

18. Duhon B, Attridge RL, Franco-Martinez AC, Maxwell PR, Hughes DW. Intravenous sodium bicarbonate therapy in severely acidotic diabetes ketoacidosis. Ann Pharmacother 47(7-8):970-5. [Epub 2013 Jun 4].

19. American Diabetes Association. Diabetes Care in the Hospital, Insulin therapy, transitioning to subcutaneous insulin 2017 Jan; 40(Supplement 1): S120-S127. https://doi. org/10.2337/dc17-S017

20. Morris LR, Murphy MB, Kitabchi AE. Bicarbonate therapy in severe diabetic ketoacidosis. Ann Intern Med 1986; 105: $836-840$ 